\title{
Factors associated with pain during intravitreal injection of anti- vascular endothelial growth factor
}

\author{
Ngoc Chau Isabelle Nguyen ${ }^{1}$ Filippo Fabro ${ }^{1} \cdot$ Aude Ambresin $^{1} \cdot$ Sakina Ezziat $^{1} \cdot$ Ciara Bergin $^{1}$.

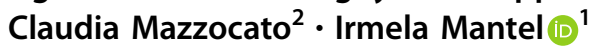

Received: 25 November 2017 / Revised: 19 March 2018 / Accepted: 22 March 2018 / Published online: 17 May 2018

(c) The Royal College of Ophthalmologists 2018

Intravitreal injection of anti-vascular endothelial growth factor (VEGF) is currently the standard-of-care treatment for various high-prevalence retinal disorders, including agerelated macular degeneration (AMD), diabetic macular oedema (DMO) and retinal vein occlusion (RVO). The injection is performed under topical anaesthesia, with some patients reporting discomfort during the injection, which could place a significant psychological burden in the long term.

According to the few reports that have investigated pain related to intraocular injections, pain scores are generally low $[1,2]$. However, the results relating to potentially associated factors, including age, gender [1], number of injections [1] and patient anxiety [2], are variable. In our study, we quantified the incidence and degree of injection-related pain, and investigated the associated risk factors.

We included 229 patients with painless retinal disease (AMD, DMO or RVO) who received intravitreal antiVEGF (ranibizumab, aflibercept or bevacizumab) injection with a $30 \mathrm{G}$ needle under topical anaesthesia (tetracaine $1 \%$ ). Before the injection, participants responded to a questionnaire about psychological pain-related elements [3]: general wellbeing, current level of anxiety, perceived quality of the information received and understanding of the treatment indication. The patients were Mean, pain during prior intravitreal injections was also asked. Each of these items was rated on a scale from 0 to 10 following the example of the Edmonton Symptom Assessment System [4]. Immediately after the procedure, participants

Irmela Mantel

irmela.mantel@fa2.ch

1 Department of Ophthalmology, University of Lausanne, Jules Gonin Eye Hospital, Fondation Asile des Aveugles,

Lausanne, Switzerland

2 Palliative and Supportive Care Service, Department of Medicine, University of Lausanne, Lausanne, Switzerland rated the degree of pain felt during the injection (scale 0 $10)$.

The results revealed pain in 132 participants (58\%), which was generally mild (mean pain score $1.7 \pm 2.0$, median 1.0), but severe (pain score 6-10) in 11 patients (5\%) (Fig. 1). Univariate analysis identified the following risk factors: preoperative anxiety $(p<0.01)$, pain during prior intravitreal injections $(p<0.01)$, chronic use of eye drops $(p=0.01)$ and quality of preoperative information provided ( $p=0.01$ ) (Table 1$)$. In multivariate analysis, the degree of anxiety $(p<0.01)$ and pain during prior injection $(p<0.01)$ were identified as independent factors (Table 1).

The role of anxiety has been reported [2], which might be related to the quality of preoperative information provided (a significant factor in univariate analysis). Measures that reduce perioperative stress (e.g. calming music) might be helpful [5]. However, we found that the surgeon's estimation of the patient's anxiety was a poor indicator of the actual anxiety level $\left(R^{2}=0.2\right)$. Thus, asking the patients about their anxiety would be more informative than relying on the surgeon's impressions.

Previous experience of painful intravitreal injections was an independent pain factor. It might be related to chronic irritation or mild subclinical inflammation of the eye. Thus, the patient's experience needs to be taken into account.

In conclusion, topical anaesthesia for intravitreal injections does not completely eliminate pain. The main independent factors are patient's anxiety and previous painful experiences. High-quality preoperative information, a reassuring surrounding and the use of relaxation technics [5] might be helpful, but this needs to be confirmed in further studies. 
Table 1 Results of the univariate and multivariate analyses of all included factors potentially associated with the degree of pain felt during the intravitreal injection procedure

\begin{tabular}{llll}
\hline Univariate analysis & $\begin{array}{l}\text { Correlation } \\
\text { coefficient }\end{array}$ & Confidence interval & Multivariate analysis \\
\hline$p$ & $\begin{array}{l}\text { Risk factor associated with } \\
\text { elevated pain }\end{array}$ & $p$
\end{tabular}

\section{Patient characteristics}

$\begin{array}{llll}\text { Age } & 0.39 & -0.01 & -0.03 \text { to } 0.01 \\ \text { Gender } & 0.15 & -0.39 & -0.91 \text { to } 0.14 \\ \text { History of intraocular surgery } & 0.89 & 0.04 & -0.49 \text { to } 0.56 \\ \begin{array}{lll}\text { Allergies } \\ \text { Allergies against drugs }\end{array} & 0.37 & 0.28 & -0.34 \text { to } 0.91 \\ \text { Other allergies } & 0.46 & 0.33 & -0.54 \text { to } 1.20 \\ \text { Any allergies } & 0.76 & 0.09 & -0.50 \text { to } 0.68\end{array}$

Systemic medications taken before injection

$\begin{array}{llll}\text { Analgesics } & 0.67 & 0.16 & -0.58 \text { to } 0.91 \\ \text { Tranquilisers } & 0.26 & 0.64 & -0.49 \text { to } 1.77\end{array}$

\section{Regular use of ocular drops}

Any ocular drops (including lubricants)

Prostaglandins

0.01 Regular use of ocular drops $\quad 0.78$

0.88
$-0.10$

0.22 to 1.34

-1.44 to 1.24

Self-reported psychological elements ranking outside the operating room (10-point scale)

$\begin{array}{lll}\text { General wellbeing } & 0.10 & 0.1 \\ \text { Level of anxiety } & 0.00 \text { Higher anxiety } & 0.1 \\ \begin{array}{l}\text { Quality of information about the } \\ \text { injection }\end{array} & 0.01 \text { Poorer information } & 0.1 \\ \begin{array}{l}\text { Appreciation and understanding } \\ \text { of treatment indication }\end{array} & 0.98 & 0.00\end{array}$

0.10

0.18

0.15

0.00

Surgeon's estimation of patient anxiety in the operating room (10-point scale)
0.74

Mean pain experienced during previous injections

\section{Redness of the eye}

Diffuse conjunctival hyperaemia

Focal episcleral hyperaemia

Ciliary hyperaemia
0.72

0.91

0.12

0.23

0.54

0.00

$-0.10$

0.07

0.02

0.27

0.00

0.23 injections

0.08 Presence of diffuse conjunctival hyperaemia

0.27

0.58
0.52

0.47

0.56
-0.02 to 0.23

0.09 to 0.28

0.00

0.03 to 0.27

-0.14 to 0.14

-0.10 to 0.14

-0.62 to 0.43

-1.15 to 1.29

-0.01 to 1.05

-1.09 to 1.62

-0.01 to 0.01

0.13 to 0.33

0.00

-0.05 to 1.09

0.41

-1.44 to 2.55

$V E G F$ vascular endothelial growth factor

\section{Compliance with ethical standards}

Conflict of interest The authors declare that they have no conflict of interest.

Informed consent Informed consent was obtained from all individual participants included in the study.
Research involving human participants All procedures performed in studies involving human participants were in accordance with the ethical standards of the institutional and/or national research committee and with the 1964 Helsinki declaration and its later amendments or comparable ethical standards. 
Fig. 1 Distribution of pain scores during the intravitreal injection procedure under topical anaesthesia, according to the patient's appreciation
Number of patients vs Score of pain

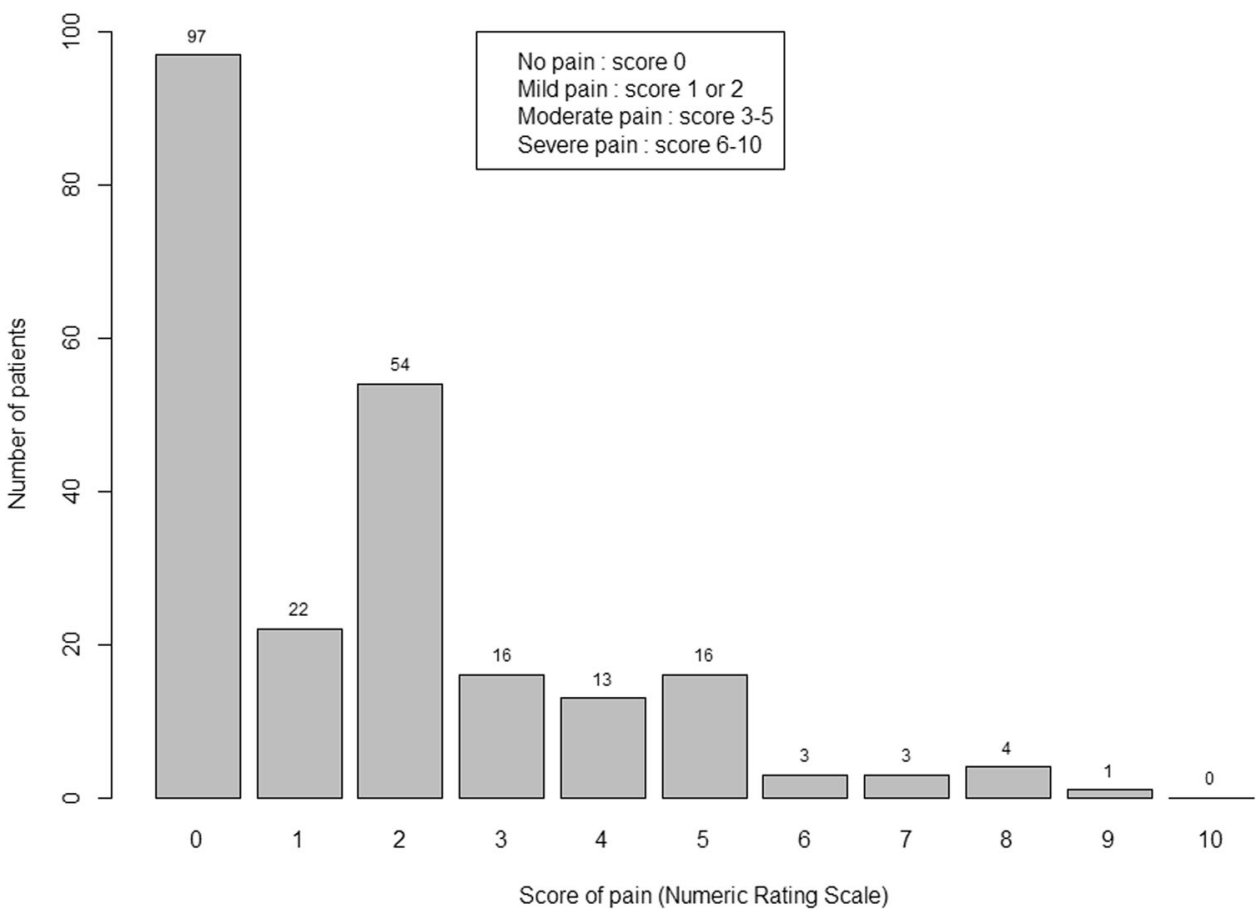

\section{References}

1. Rifkin L, Schaal S. Factors affecting patients' pain intensity during in office intravitreal injection procedure. Retina. 2012;32:696-700.

2. Segal O, Segal-Trivitz Y, Nemet AY, Cohen P, Geffen N, Mimouni M. Anxiety levels and perceived pain intensity during intravitreal injections. Acta Ophthalmol. 2016;94:203-4.

3. Okamura H, Fukui S, Nagasaka Y, Koike M, Uchitomi Y. Psychoeducational intervention for patients with primary breast cancer and patient satisfaction with information: an exploratory analysis. Breast Cancer Res Treat. 2003;80:331-8.

4. Nekolaichuk C, Watanabe S, Beaumont C. The Edmonton Symptom Assessment System: a 15-year retrospective review of validation studies (1991--2006). Palliat Med. 2008;22:111-22.

5. van der Heijden MJ, Oliai Araghi S, van Dijk M, Jeekel J, Hunink MG. The effects of perioperative music interventions in pediatric surgery: a systematic review and meta-analysis of randomized controlled trials. PLoS ONE. 2015;10:e133608.

\title{
Management of anterior capsular contraction syndrome: pitfall of circular capsulotomy technique with the neodymium YAG laser
}

\author{
Craig Wilde $^{1} \cdot$ Andrew Ross $^{1} \cdot$ Mary Awad ${ }^{1} \cdot$ Hean-Choon Chen ${ }^{2} \cdot$ Harminder S Dua $\mathbb{D}^{1}$
}

Received: 7 March 2018 / Accepted: 15 March 2018 / Published online: 18 May 2018

(c) The Royal College of Ophthalmologists 2018

\section{Harminder S Dua}

Harminder.Dua@nottingham.ac.uk

1 Ophthalmology and Vision Sciences, Division of Clinical Neurosciences, B Floor, EENT Centre, Queen's Medical Centre, University of Nottingham, Nottingham, UK

2 Royal Derby Hospital, Uttoxeter Road, Derby DE22 3NE, UK
Dear Editor,

Capsular contraction syndrome (CCS) is a distinct clinical entity associated with continuous curvilinear lcapsulorhexis (CCC) performed during phacoemulsificaion [1]. Although some degree of anterior capsular whitening and 